\title{
Adverse Drug Reaction Reporting Pattern in Turkey: Analysis of the National Database in the Context of the First Pharmacovigilance Legislation
}

\author{
Gulnihal Ozcan $^{1} \cdot$ Emel Aykac $^{1} \cdot$ Yelda Kasap $^{1} \cdot$ Nergiz T. Nemutlu $^{1} \cdot$ \\ Ebru Sen ${ }^{1}$ - N. Demet Aydinkarahaliloglu'
}

Published online: 8 January 2016

(c) The Author(s) 2016. This article is published with open access at Springerlink.com

\begin{abstract}
Introduction In Turkey, pharmacovigilance began in 1985. A fully structured adverse drug reaction (ADR)-reporting system was established with the publication of the first pharmacovigilance regulation in 2005. Subsequent regulation published in 2014 brought further improvements to the system.

Objective In this study, we aimed to analyse the ADRreporting pattern in the context of the first pharmacovigilance legislation in Turkey.

Methods We analysed ADR reports submitted to the Turkish Pharmacovigilance Center (TUFAM) from 2005 to 2014 with respect to reporting rate (RR), patient characteristics, type of the ADRs, suspected drugs, source of the report and the profession of the reporter.

Results The annual RR increased gradually over the study period. RRs for females were greater than those for males. RRs were highly correlated with age. Most commonly reported ADRs were skin and subcutaneous tissue disorders. Most commonly suspected drugs were antineoplastic and immunomodulating agents. There was no remarkable change in reporting pattern of ADRs, patient characteristics or classes of suspected drugs over the years. The most common source of reports was spontaneous reporting. Contribution of the reports from studies
\end{abstract}

Electronic supplementary material The online version of this article (doi:10.1007/s40801-015-0054-1) contains supplementary material, which is available to authorized users.

N. Demet Aydinkarahaliloglu

demet.aydink@titck.gov.tr

1 Department of Risk Management, Turkish

Pharmacovigilance Center (TUFAM), Turkish Medicines and Medical Devices Agency, 06520 Ankara, Turkey increased gradually. Most of the reports were reported by physicians. RRs by pharmacists increased substantially over the years.

Conclusion This study showed that the annual RR increased gradually over the 9-year study period. This increase was neither due to an increased reporting of a specific group of ADRs or drugs, nor to an increased reporting in a specific group of patients. There was a general increase in RR in parallel to pharmacovigilance activities.

\section{Key Points}

Reporting rate of adverse drug reactions increased gradually over the years in Turkey in parallel to awareness activities on drug safety.

In Turkey, the reporting rate of adverse drug reactions was higher in females and elderly patients.

The number of ADRs reported per million boxes of drug consumption was highest for antineoplastic and immunomodulating agents.

\section{Introduction}

Adverse drug reactions (ADRs) are common causes of mortality and morbidity all around the world $[1,2]$. They represent an important economic burden for health systems $[3,4]$. ADRs that occur in real-world medical practice cannot always be predicted by pre-marketing data since a limited number of selected patients are enrolled in clinical trials for specific indications and monitored for a limited period of time. Therefore, post-marketing surveillance is 
the most important tool for pharmacovigilance systems for early detection of unexpected and serious ADRs [5].

World data on ADRs are collected at the Uppsala Monitoring Center (UMC) constituted under the auspices of the World Health Organization's (WHO) Programme for International Drug Monitoring [5, 6]. All member countries send national ADR reports to the UMCs individual case safety reports (ICSR) database system, VigiBase [7, 8]. UMC continuously monitors the VigiBase for possible signals and alerts. Alerts from UMC constitute an important reference for decision-making processes of national pharmacovigilance authorities. However, ADR profiles vary from country to country owing to differences in genetics, diet and traditions of populations, and medical practices [5, $6,9]$. Additionally, pharmacovigilance legislations and the structure of the pharmacovigilance systems vary among WHO member countries $[10,11]$. Because of these factors, information derived from the cumulative data may not always be relevant or applicable to individual populations. To be able to detect local signals and take accurate actions for minimization of the risk, it is important for countries to monitor and analyse their own national ADR databases continually. Such analysis can also guide actions to stimulate ADR reporting, and help to assess the effectivity of national legislations and pharmacovigilance activities.

In Turkey, pharmacovigilance activities started in 1985 with the establishment of the "Turkish Adverse Drug Reaction Monitoring and Evaluation Center" (TADMER) under the General Directorate of Pharmaceuticals and Pharmacy. In 1987, TADMER joined the WHO Programme as an official member. In 2005, first pharmacovigilance regulation, "Regulation on the Monitoring and Assessment of the Safety of Medicinal Products for Human Use", became effective [12]. With this regulation, TADMER started to conduct pharmacovigilance activities under the name "Turkish Pharmacovigilance Center" (TUFAM), in order to stress the term "Pharmacovigilance". In the regulation, major responsibilities of TUFAM were defined as: monitoring national ADR reports and drug safety alerts worldwide, communicating drug safety alerts to healthcare professionals, educating physicians and pharmacists on pharmacovigilance, conducting risk minimization methods, and assessing conformity of risk management plans and periodic safety update reports. Taking this first regulation as reference, responsibilities of Authorization Holders, Pharmacovigilance Inspections and Structure of Risk Management Systems were addressed in detail in the guidelines published in 2005, 2009 and 2011, respectively. In 2012, the General Directorate of Pharmaceuticals and Pharmacy became an agency called the Turkish Medicines and Medical Devices Agency. With this structural change, a Risk Management Unit was formed to take over risk minimization activities. Since then, TUFAM has been concentrating on monitoring and assessing national ADR reports.

National ADR reports reach TUFAM from two major sources: healthcare professionals and marketing authorization holders (MAHs). Healthcare professionals can notify spontaneous reports to the TUFAM either directly or by means of the pharmacovigilance contact points (PvCPs) within the health organization that they are employed in. PvCPs are physicians or pharmacists who are responsible for encouraging the notification of ADRs, collecting and communicating information to TUFAM, and carrying out training and awareness activities at hospitals they work in. According to regulation, a PvCP should be assigned to work at university hospitals, training and research hospitals, and private hospitals with a bed capacity of 50 or more. This regulation was later expanded to cover all hospitals. This method is different from many countries, and has the intention of communicating information faster between TUFAM and health-care professionals.

MAHs are responsible for keeping the records of all suspected ADRs and notifying serious ADRs occurring in Turkey to the TUFAM within 15 days. They collect both spontaneous reports and solicited reports from patient support programmes where they receive and collect information relating to the use of their medicinal products. In Turkey, MAHs are also responsible for screening national and international literature for ADRs regarding the local population and forwarding a copy to TUFAM.

Spontaneous and solicited ADR reports reaching TUFAM from healthcare professionals and MAHs are sent to the VigiBase as ICSRs. In this way TUFAM contributes to the integration of world data on ADRs as intended by the WHO programme. In 2014, national pharmacovigilance regulation was revised in the context of harmonization with EU directives [13]. With these new regulations, patient reports also started to be accepted and sent to the VigiBase by TUFAM. Additionally national ADRs mentioned in the literature started to be sent to the Vigibase if they complied with the requirements of an ICSR.

In this study, we aimed to analyse the national ADR reports submitted to VigiBase in the period for which the first pharmacovigilance legislation was effective and present the ADR reporting pattern in Turkey. This is the first detailed study on the National ADR Database of Turkey.

\section{Methods}

\subsection{Data}

This study included national ADR reports for all the marketed drugs that were submitted to VigiBase by TUFAM between 1 July 2005 and 31 December 2013. In the study 
period all of the spontaneous and solicited reports that reach TUFAM and satisfy the minimum criteria of reporting were submitted to Vigibase. Minimum criteria of reporting were defined in the regulation as: (a) an identifiable reporter, (b) an identifiable patient, (c) at least one suspected drug, and (d) at least one ADR. The reports for which missing information could not be completed were not submitted to the VigiBase, since they did not comply with a valid ICSR.

For each report, information about the type of the report, qualification of the reporter, age and sex of the patient, suspected medications, ADRs and seriousness of the ADRs was extracted from the VigiBase. Number of reports in individual categories was counted for each study-year. Data were presented as report count, percentage of reports and/or RR for individual groups. RR was used as a measure of annual frequency of reporting in each category and estimated by adjusting the number of reports in the individual category in a year by the number of individuals residing in the corresponding category for that year. Different from other years, data for 2005 were given only for the second half of the year since the first pharmacovigilance regulation became effective on 30 June 2005.

RRs for specific age or sex groups were estimated to observe the differences in RRs within age or sex groups. The Turkish Statistical Institute's population by sex and age groups data were used for estimation [14]. Additionally, RRs per million patient visits and million inpatients were estimated as a measure of frequency of reporting for a million visits and a million hospitalizations. RRs for a million boxes of drug consumption and a thousand individuals in health profession groups were estimated to investigate the pattern of reporting for different drug and reporter groups, respectively. The Turkish Ministry of Health's data on number of hospital visits by years, number of inpatients by years, consumption of drugs by years and number of healthcare professionals by years were used for estimation [15]. Estimation was done only for the years in which Ministry of Health's data are available.

\subsection{Classification of Reports}

In Vigibase there are two categories for defining the type of report: spontaneous reports and reports from studies. The terminology for spontaneous reports in our database and Vigibase is the same but solicited reports from patient support programmes in our database were coded as reports from studies during entry into the Vigibase.

Reporters were classified according to six groups: physicians, pharmacists, other health professionals, consumers, lawyers and unknown.

For age grouping 5-year interval age groups were used. Accordingly, patients were divided into the following age groups: $0-4,5-9,10-14,15-19,20-24,25-29,30-34$, $35-39,40-44,45-49,50-54,55-59,60-64,65-69,70-74$ and +75 years. In order to calculate age-specific RRs, the number of ADR reports within each 5-year interval age group was adjusted by the number of individuals in the age group for the corresponding year [14].

ADRs were classified according to the Medical Dictionary for Regulatory Activities (MedDRA) System Organ Class (SOC) [16]. Seriousness of ADRs was classified according to the International Conference on Harmonization of Technical Requirements for Registration of Pharmaceuticals for Human Use (ICH) E2A criteria used in VigiBase [17].

Suspected drugs were classified according to the Anatomical Therapeutic Chemical (ATC) Classification system at level 2 at most, which provides information about pharmacological/therapeutic subgroup [18].

\subsection{Statistics}

Student's $t$ test was used for analysing the significance of difference between RRs for a million inhabitants and a million inpatients per year, and the difference between RRs in sex groups. Pearson's correlation coefficient $(r)$ was calculated for correlation analysis.

\section{Results}

We examined spontaneous and solicited reports that were submitted to TUFAM for all the marketed drugs in the period from June 2005 to the end of 2013. During the study period, a total of 8065 reports satisfied the minimum criteria for reporting and were thus included in the study.

\subsection{Adverse Drug Reaction (ADR) Reporting Rates}

The number of ADR reports submitted in a year increased gradually over the study period (Fig. 1).

An increase in population, hospital visits or drug consumption may cause bias in favour of an increase in reporting of ADRs over years. To address these possibilities we adjusted the number of reports with the number of inhabitants (in millions), patient visits (in millions) and boxes of drug consumed (in millions) in a year, and estimated annual RRs (Table 1). We observed that the annual RR for a million inhabitants increased from 1.5 in 2005 to 32.1 in 2013 in parallel with the annual number of reports. The greatest increase in RR was observed in 2012 with a 10.7 increase. The annual RR for a million patient visits and a million boxes of drug consumption also increased over the years, and were highly correlated with the increase in RR for a million inhabitants $(r=0.99)$. Additionally, we observed that the annual RRs for a million inhabitants 




Fig. 1 Annual number of adverse drug reaction (ADR) reports submitted to Vigibase by the Turkish Pharmacovigilance Centre (TUFAM) between 2005 and 2013. Asterisk data was given for the second half of the year

were significantly higher than those for a million patient visits $(p<0.05)$.

\subsection{Type of Reports}

The most common type of reporting was spontaneous reports in all years investigated (Table 2). Contribution of reports from studies increased gradually starting from 2010. The greatest increase in reporting from studies was observed in 2012.

\subsection{ADRs by Sex}

Overall, $56.5 \%$ of reports were reported for females. The percentage of reports for females was greater than that for males for all years (data not shown). When the number of reports for sex groups was standardized for the corresponding population in millions, annual RRs for females were still significantly greater than those for males $(p<0.05)$ (Table 3).

\subsection{ADRs by Age}

We calculated the RRs per million inhabitants in 5-year age group intervals to observe the pattern of reporting with respect to age groups (Fig. 2). The 9-year averages of RRs were highest in age groups 65-69, 70-74, 60-64 and +75 years, and tended to decrease with the decrease in age of the patients. Despite the high positive correlation between RRs and age ( $r=0.93$ ), we observed a higher RR in 0-4 years age group compared to adjacent age groups. When this age group was removed from the correlation analysis, strength of correlation increased further $(r=0.97)$.

\subsection{Type of Reporter}

In general, most of the ADR reports were reported by physicians $(59.8 \%)$, followed by other health professionals $(28.7 \%)$ and pharmacists $(9.1 \%)$. A small percentage of reports was reported by consumers $(2.3 \%$ ) (data not shown).

In Table 4, RRs are given that were calculated by adjusting the number of reports with the number of actively working professionals (in thousands) in the corresponding year. In general, RRs of physicians were greater than those of pharmacists. However, RRs of pharmacists increased
Table 1 Annual reporting rate of adverse drug reactions per million inhabitants, million patient visits and million boxes of drug consumption in Turkey between 2005 and 2013

\begin{tabular}{lccccccccc}
\hline Year & $2005^{\mathrm{a}}$ & 2006 & 2007 & 2008 & 2009 & 2010 & 2011 & 2012 & 2013 \\
\hline $\mathrm{RR}^{\mathrm{b}}$ (\#/mill. inhab./year) & 1.5 & 4.3 & 5.0 & 5.4 & 7.1 & 13.5 & 14.6 & 25.3 & 32.1 \\
Rate of increase in RR $^{\mathrm{b}}$ & & 2.8 & 0.7 & 0.4 & 1.7 & 6.4 & 1.1 & 10.7 & 6.8 \\
$\mathrm{RR}^{\mathrm{c}}$ (\#/mill. visits/year) & & & & & 1.8 & 3.3 & 3.2 & 5.4 & 6.5 \\
$\mathrm{RR}^{\mathrm{d}}$ (\#/mill. boxes /year) & & & 0.3 & 0.3 & 0.4 & 0.7 & 0.7 & 1.1 & 1.6 \\
\hline
\end{tabular}

$R R$ reporting rate

${ }^{a}$ Data was given for the second half of the year only

b Number of reports/million inhabitants/year

c Number of reports/million patient visits/year

${ }^{\mathrm{d}}$ Number of reports/million boxes of drug consumption/year

\begin{tabular}{lrrrrrrrrr}
\hline Year & $2005^{\mathrm{a}}$ & 2006 & 2007 & 2008 & 2009 & 2010 & 2011 & 2012 & 2013 \\
\hline Spontaneous & 100.0 & 99.0 & 100.0 & 99.5 & 99.6 & 92.3 & 81.8 & 71.1 & 74.5 \\
Report from study & 0.0 & 1.0 & 0.0 & 0.5 & 0.4 & 7.7 & 18.2 & 28.9 & 25.5 \\
Total & 100.0 & 100.0 & 100.0 & 100.0 & 100.0 & 100.0 & 100.0 & 100.0 & 100.0 \\
\hline
\end{tabular}

${ }^{a}$ Data were given for the second half of the year
Table 2 Percentage distribution of adverse drug reaction reports by type in Turkey between 2005 and 2013 
Table 3 Annual reporting rate of adverse drug reactions for females and males in Turkey between 2005 and 2013

\begin{tabular}{lccccccccc}
\hline Year & $2005^{\mathrm{a}}$ & 2006 & 2007 & 2008 & 2009 & 2010 & 2011 & 2012 & 2013 \\
\hline Reporting rate & & & & & & & & & \\
Female & 2.2 & 4.8 & 5.5 & 6.1 & 8.0 & 15.2 & 17.6 & 28.5 & 35.7 \\
Male & 0.8 & 3.7 & 4.3 & 4.7 & 6.0 & 11.5 & 11.2 & 20.7 & 26.7 \\
\hline
\end{tabular}

a Data were given for the second half of the year

b Number of reports/million individuals in the sex group/year



Fig. 2 Reporting rate (RR) of adverse drug reactions by 5-year interval age groups in Turkey between 2005 and 2013 (9-year average)

Table 4 Annual reporting rate of adverse drug reactions by physicians and pharmacists in Turkey between 2009 and 2013

\begin{tabular}{lllllr}
\hline Health professional & 2009 & 2010 & 2011 & 2012 & 2013 \\
\hline Physician & 3.6 & 6.2 & 5.5 & 6.7 & 8.0 \\
Pharmacist & 1.9 & 2.9 & 4.3 & 6.6 & 10.3 \\
\hline
\end{tabular}

Number of reports/thousand professionals actively working in Turkey/year

substantially over the years and even exceeded the RRs for physicians in the year 2013. RRs of other health professionals could not be calculated since the definition of "other health professionals" used in the Health Statistics in Turkey is more comprehensive, and included: surgery technician, biologist, environmental health technician, child development specialist, dental technician, dietitian, physical therapy technician, physiotherapist, first aid and emergency care repairman, heart-lung pump operation technician, laboratory repairman, laboratory technician, audiologist, audiometric repairman, audiometric technician, orthopaedic technician, pathological anatomy technician, perfusion pump technician, prosthetics technician, psychologist, radiographer, health physicist, war health officer, health technician, health repairman, cytopathologist, social worker, medical secretary, medical technologist and public health technician in addition to nurse, anaesthesia technician, and emergency and first aid technician included in national pharmacovigilance system.

\subsection{ADRs According to Seriousness}

In the study period a total of 16,248 ADRs were reported in 8065 reports. The average number of ADRs per report showed a slight increase from 1.8 in 2005 to 2.3 in 2013 with a 9-year average of 2.0 (data not shown).

Among all the reports, $70.5 \%$ included a serious ADR. The most commonly specified seriousness criterion was medically important conditions (44.8\%), which was followed by hospitalization or prolongation of existing hospitalization (36.6\%), life-threatening conditions (14.3\%), death $(8.6 \%)$, persistent or significant disability/incapacity $(2.7 \%)$ and congenital anomalies $(0.2 \%)$ (data not shown).

We wanted to estimate the frequency of reporting of ADRs as a cause of hospitalization or prolongation of existing hospitalization among hospitalized patients. We observed a gradual increase in RR over years with a 32.5 increase per million inpatients from 2009 to 2013 (Fig. 3).

During the study period, seriousness criterion was reported as congenital anomaly in 14 reports. Suspected drugs and ADR terms for these reports are listed in Table 5.

\subsection{ADRs by System Organ Classification (SOC)}

The percentage distribution of most frequently reported ADRs by SOC is given in Fig. 4. These 14 SOCs listed in the figure covered $90.2 \%$ of all ADRs in the database.

Skin and subcutaneous tissue disorders, general disorders and administration site conditions, gastrointestinal disorders and nervous system disorders were the most frequently reported ADR SOCs, constituting together approximately $50 \%$ of ADR SOCs in the database (Fig. 4). There was no remarkable change in reporting pattern of ADRs by SOCs over time. These four SOCs were in the top five of most frequently reported ADR SOCs in all years investigated (data not shown).

\subsection{ADRs by Therapeutic Groups}

In the study period a total of 9394 drugs were reported in 8065 reports. In $88.8 \%$ of the reports only one drug was reported as the suspected drug. In the rest of the reports 
mostly two drugs $(6.8 \%)$ or three drugs $(3.9 \%)$ were suspected to cause the ADR. The average number of drugs per report was around 1.2 for all years.

Most commonly reported drugs belong to the ATC classes antineoplastic and immunomodulating agents $(26.5 \%)$, anti-infectives for systemic use $(24.5 \%)$ and nervous system drugs (14.3\%) (Fig. 5). These three ATC classes were the most frequently reported drug groups in all years investigated (data not shown) and covered $65.5 \%$ of all the suspected drugs reported in the 9-year period.

Reporting rate with respect to million boxes of drug consumption slightly increased over the study period

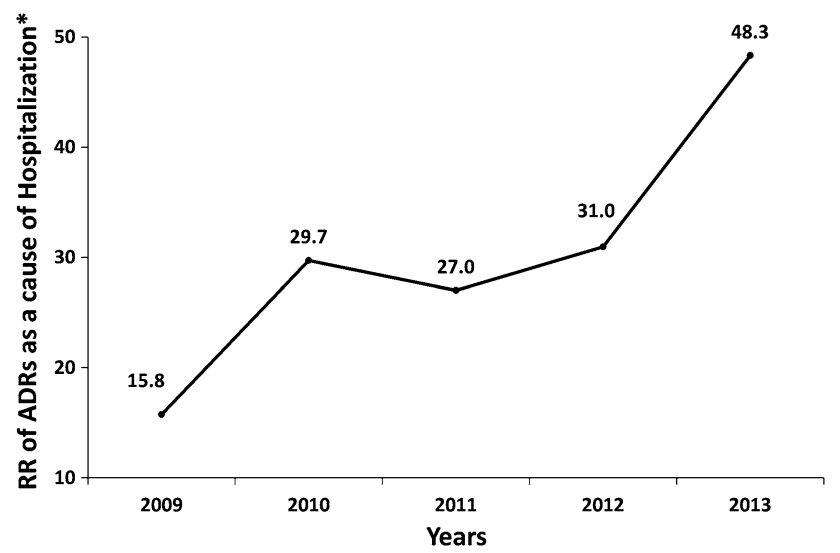

Fig. 3 Annual reporting rate (RR) of adverse drug reactions as a cause of hospitalization or prolongation of hospitalization between 2009 and 2013. Asterisk number of reports in which hospitalization or prolongation of hospitalization was reported as the seriousness criterion /number of inpatients in Turkey/year
(Table 1). RR for antineoplastic and immunomodulating agents was significantly high compared to overall RR $(p<0.01)$ (Supplementary Table 1$)$. With the second-level ATC subgroups, most commonly reported drug groups were antibacterials for systemic use, immunosuppressants and antineoplastic agents (Supplementary Table 2). Most commonly reported active substances and percentage of serious ADRs reported for these drugs were listed in Table 6.

ADR reports related to vaccines constitute a very small percentage of the database $(1.46 \%, 118$ reports) since safety of vaccines has being monitored by immunization programmes in Turkey. Most commonly reported vaccines in our database were pneumococcal conjugate vaccine (40 reports), rotavirus vaccine (19 reports) and influenza vaccines (19 reports) in the 9-year study period.

\section{Discussion}

\subsection{ADR Reporting Rates and Report Types}

This is the first study to analyse general ADR reporting patterns in the national pharmacovigilance database of Turkey. Our analyses showed that ADR RRs for a million inhabitants in Turkey increased remarkably over the last 9 years (Table 1). Most remarkable increases were observed in 2010, 2012 and 2013. There were salient advances in these years likely to contribute to the increased RR. In 2010, patient support programmes became effective

Table 5 Suspected drugs and adverse drug reaction (ADR) terms in the reports where congenital anomaly was reported as the seriousness criterion in Turkey between 2005 and 2013

\begin{tabular}{lll}
\hline Case & Suspected drug & ADR terms \\
\hline 1 & Adalimumab & Maternal exposure during pregnancy; abortion \\
2 & Adefovir dipivoxil & Congenital anomaly, third finger first phalanx deficiency \\
3 & Butamirate citrate & Dysmorphism; alopecia areata; hypotonia; mental retardation; failure to thrive; brain \\
& & malformation; congenital deafness; congenital blindness \\
4 & Dasatinib & Flatulence; maternal exposure during pregnancy \\
5 & Drospirenone/ethinylestradiol & Therapeutic abortion due to anomaly \\
6 & Entecavir & Congenital musculoskeletal anomaly \\
7 & Escitalopram, mirtazapine, hyoscine $n-$ & Maternal exposure during pregnancy; congenital anomaly not otherwise specified, \\
& butylbromide, medazepam & abnormality of right forearm reduction of the fetus \\
8 & Insulin aspart & Congenital anomaly (5-alfa reductase enzyme deficiency dependent ambiguous genitalia, \\
& & left coronary artery variation anomaly and horse-shoe kidney) \\
9 & Insulin regular/insulin isophane & Maternal exposure during pregnancy; congenital hand malformation; limb malformation \\
10 & Insulin regular/insulin isophane & Premature baby; breech presentation; fetal exposure during pregnancy \\
11 & Isotretinoin & Congenital hydrocephalus; eyelid ptosis; cleft palate; ear malformation \\
12 & Olanzapine & Maternal exposure during pregnancy; hypotonia neonatal; cyanosis neonatal; talipes \\
13 & Ranibizumab & Ventricular septal defect \\
14 & Tenofovir disoproxil fumarate & Premature baby; fetal death; placental disorder \\
\hline
\end{tabular}


Fig. 4 Percentage distribution of adverse drug reactions by most frequently reported system organ classes in Turkey between 2005 and 2013

Fig. 5 Percentage distribution of adverse drug reaction reports by Anatomical Therapeutic Chemical (ATC) class (first level) of suspected drugs in Turkey between 2005 and 2013

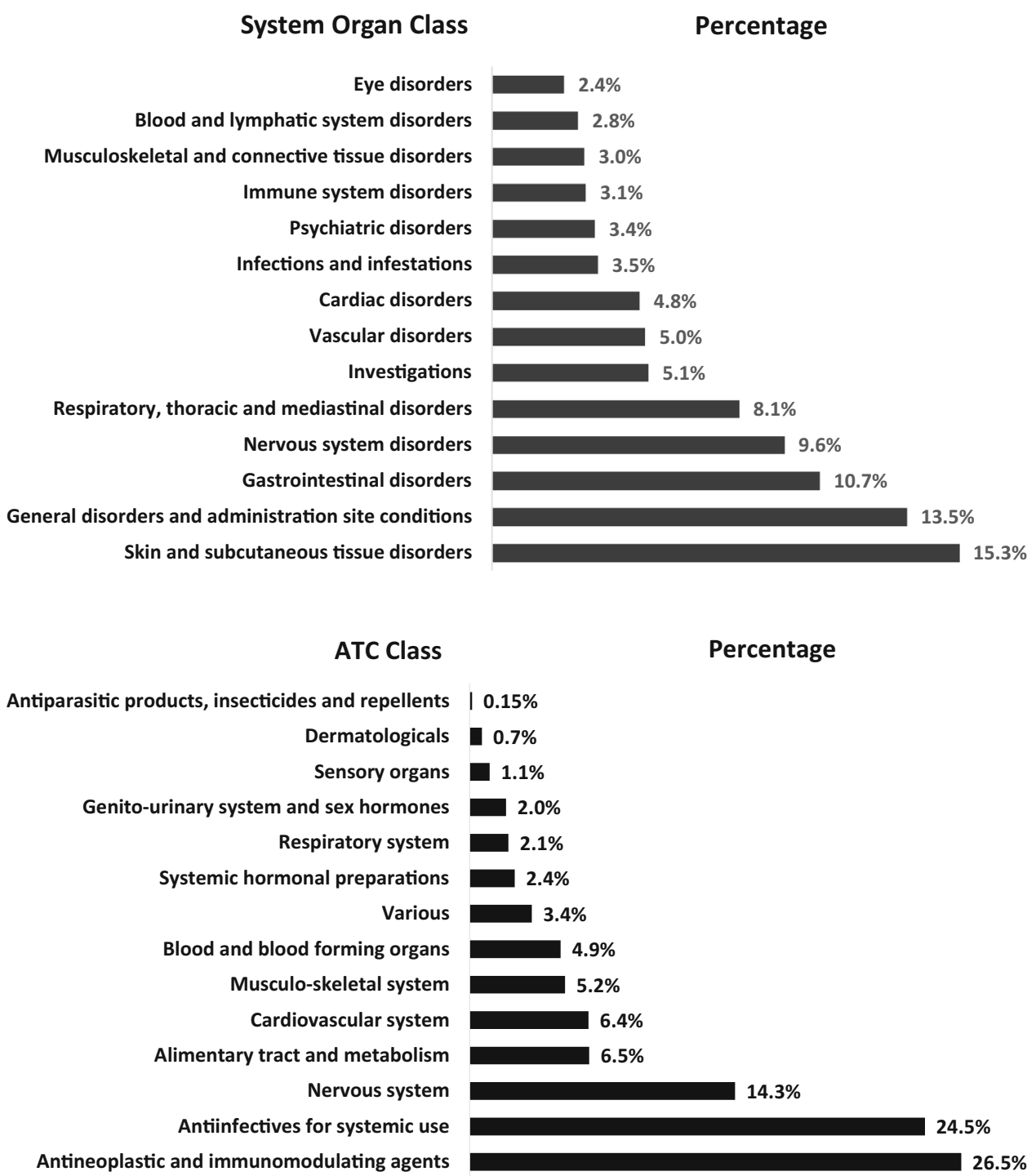

System Organ Class

Eye disorders

Psychiatric disorders

and infestations

scular disorders

General disorders and administration site conditions

Skin and subcutaneous tissue disorders
Table 6 Report counts and Anatomical Therapeutic Chemical (ATC) codes for most commonly reported active substances in Turkey between 2005 and 2013 and percentage of serious adverse drug reactions (ADRs) for each substance

\begin{tabular}{lllcc}
\hline & Suspected drug & Report count & Percent of serious ADRs & ATC code \\
\hline 1 & Adalimumab & 457 & 98 & L04 \\
2 & Interferon beta-1b & 289 & 74 & L03 \\
3 & Etanercept & 230 & 59 & L04 \\
4 & Ceftriaxone sodium & 214 & 32 & J01 \\
5 & Peginterferon alfa & 210 & 96 & L03 \\
6 & Ribavirin & 209 & 98 & J05 \\
7 & Telaprevir & 196 & 100 & J05 \\
8 & Infliximab & 147 & 61 & L04 \\
9 & Moxifloxacin & 138 & 26 & J01 \\
10 & Cefazolin sodium & 106 & & J01 \\
\hline
\end{tabular}

and contribution of these programmes to the ADR database increased after that (Table 2). With the structural change in our National Pharmacovigilance Center in 2012, TUFAM started to concentrate mostly on monitoring and assessment of national ADR reports and was given the opportunity to dedicate more resources on the activities to raise awareness 
on pharmacovigilance and encourage ADR reporting nationwide. In 2012, a remarkable increase was observed in the rate of reporting from both patient support programmes and spontaneous sources. This observation can be evaluated as a translation of the effectivity of the structural change. In 2013, TUFAM started to accept patient notifications in the context of a pilot project via an electronic system developed by UMC. The system was incorporated into the Turkish Medicines and Medical Devices Agency's website. In the first year of the project, a remarkable number of reports were submitted by consumers and contributed to the increasing trend in annual RR (data not shown).

In the study, we observed a significantly higher annual RR for million inhabitants than that for million patient visits. This observation suggests a considerable frequency of ADRs related to self-medication. Self-medication is a public health problem all over the world and studies have shown that self-medication-related ADRs should not be ignored [19, 20]. This point should also be addressed in Turkey by future studies.

\subsection{ADRs by Sex and Age}

In the study period RR for female patients was significantly high than that for males (Table 3). This is consistent with other studies [21-23]. There can be different factors contributing to the higher rate of reporting in females: the incidence of ADRs may be higher in females, hospital visits may more frequently involve female patients or female patients may more frequently consult a healthcare professional concerning an ADR. However, it is not possible to discern the individual contribution of these factors to the existing data.

In accordance with the results of previous studies [21, 23, 24], RRs were higher in the elderly where drug consumption is high (Fig. 2). Interestingly, RRs for the 0-4 years age group was remarkably high compared to those of adjacent age groups. In the study period, a patient support programme regarding use of palivizumab was active. Since palivizumab is used mostly in neonates, a large number of reports that reached TUFAM from the palivizumab patient support programme in the study period could be an explanation for the relatively high RR in the 0-4 year age group.

\subsection{ADRs by Type of Reporter}

Reports from physicians constituted the greatest percentage of reports, followed by reports from other health professionals and pharmacists. However, these data does not reflect reporting behaviors of profession groups since number of individuals in each profession group vary greatly. Therefore, number of reports was adjusted with the number of individuals in each profession group for the years between 2009 and 2013 in which corresponding data are available. It was observed that RR of physicians was greater than that of pharmacists for the years 2009-2012. However, RR of pharmacists increased substantially over the years and exceeded the RR of physicians in 2013 (Table 4). This increase in RR of pharmacists may be explained by the fact that mostly pharmacists being employed as PvCPs.

\subsection{ADRs by Seriousness}

Overall, $70.5 \%$ of the reports included a serious ADR. This is expected since there is emphasis on reporting of serious ADRs in pharmacovigilance regulations in Turkey. Reporting of hospitalization or prolongation of existing hospitalization as seriousness criteria increased gradually over time and reached 48.3 per million inpatients in 2013 (Fig. 3). This may be a reflection of the increased awareness regarding ADRs and the overall increase in RRs.

During the study period, the seriousness criterion was reported as congenital anomaly in 14 reports (Table 5). In two of the reports the suspected drugs were drospirenone/ ethinylestradiol and isotretinoin which have a pregnancy category of $\mathrm{X}$. In the first report, "therapeutic abortion due to anomaly" was reported with exposure to drospirenone/ ethinylestradiol (Case 5). In the literature there are reports that relate oral contraceptive use to malformations of the genitals in male infants [25, 26]. However, in the report mentioned above, the anomaly was not specified. In the second report (Case 11), "Congenital hydrocephalus; Eyelid ptosis; Cleft palate; Ear malformation" were reported which are well-defined congenital anomalies related to isotretinoin exposure during pregnancy [27-29]. Two out of 14 reports concern drugs with a pregnancy category of D, dasatinib and ranibizumab. For dasatinib, congenital anomaly was not specified in the report (Case 4) but in the product information hydrops fetalis, fetal leukopenia and fetal thrombocytopenia were reported and the possibility of congenital malformations, including neural tube defects, were mentioned [30]. For ranibizumab, ventricular septal defect was reported (Case 13). As far as we know, there is no such case in the literature. Among the suspected drugs with pregnancy category $\mathrm{C}$, only adefovir and olanzapine had been mentioned in congenital anomaly cases in the literature. Congenital heart defect was reported with paternal administration of adefovir in the literature [31], which is not associated with "phalanx deficiency" reported in our case (Case 2). An increased risk of neonatal extrapyramidal and/or withdrawal symptoms (e.g., agitation, hypertonia, hypotonia, tremor, somnolence, respiratory distress and feeding disorder) were reported to be associated with exposure to antipsychotic drugs during the 
third trimester of pregnancy [32-34]. Neonatal hypotonia and cyanosis reported for olanzapine in our database (Case 12 ) is compatible with withdrawal symptoms. Additionally "talipes" was observed in the case. Although a specific pattern of fetal limb or organ malformation have not been reported to be related to antipsychotics, there is a case report where hip dysplasia was observed after olanzapine exposure during pregnancy [35]. Other drugs listed in Table 5 do not appear to be associated with an increased risk for major malformations. Interestingly, three congenital anomaly cases were reported for insulin analogs (Case 8-10) which are preferred during pregnancy owing to their inability to pass transplacentally. Congenital anomalies reported in these cases were: (1) 5-alfa reductase enzyme deficiency dependent ambiguous genitalia, left coronary artery variation anomaly and horse-shoe kidney, (2) congenital hand malformation and limb malformation, and (3) premature baby and breech presentation. The high relative reporting rate for this therapeutic group might be considered a coincidence since pregnancy exposure registries and patient support programmes have being conducted for use of insulin analogues during pregnancy in Turkey.

\subsection{ADRs by System Organ Classification}

Among 36 SOCs for ADRs, skin and subcutaneous tissue disorders, general disorders and administration site conditions, gastrointestinal disorders, and nervous system disorders were the most frequently reported ADR SOCs covering approximately $50 \%$ of total ADRs (Fig. 4). This pattern is similar to the global pattern of ADRs between the years 2000-2009, where general disorders and administration site conditions, skin and subcutaneous tissue disorders, nervous system disorders and gastrointestinal disorders were the most frequently reported SOCs for ADRs [10].

\subsection{ADRs by Therapeutic Groups}

In the majority of the reports, only one drug was reported as the suspected drug. RRs per million boxes of drug consumption increased slightly over the study period (Table 1). Similar to patterns of ADRs in upper-middle income countries reported by Aagard et al. [10], drugs from ATC classes of "antiinfectives for systemic use" (24.5\%) and "nervous system" (14.4\%) had high rates of reporting in Turkey (Fig. 5). However, "Antineoplastic and immunomodulating agents" (26.5\%) was the most frequently reported ATC drug group in Turkey; although it was the eighth most common ATC group in the uppermiddle income countries [10]. It should be noticed that the study by Aagard et al. analysed only spontaneous reports submitted to VigiBase from 2000 to 2009, whereas our study included both spontaneous reports and reports from studies submitted between 2005 and 2013. These differences in the inclusion criteria for report type and the study periods may be the reason for the difference in rank of reporting for ATC groups. Differences in the characteristics of consumption of these drugs could also be a factor affecting the frequency of reporting in each country. However, number of reports adjusted by the consumption of million boxes of drugs in Turkey was still highest for antineoplastic and immunomodulating agents (Supplementary Table 1). Estimated RRs for this ATC group even reached to 77 times the overall reporting rate in 2011. The most probable explanation for this remarkably high RR is the high number of antineoplastic and immunomodulating agents involved in patient support programmes in Turkey. Examples for these drugs include interferon, eculizumab, fingolimod, adalimumab, etanercept, golimumab, infliximab, tacrolimus and lenalidomide which are highly represented in the database with solicited reports from patient support programmes. Among these, five drugs, namely adalimumab, interferon, etanercept, peginterferon and infliximab were in the list of ten most commonly reported drugs over the study period (Table 6). Percentage of serious ADRs reported with these drugs was generally higher than the overall percentage of serious ADRs in the database. This may be a reflection of the legislation, since MAHs are responsible for notifying serious ADR reports to TUFAM. Accordingly antivirals, Ribavirin and Telaprevir which are also included in patient support programs are represented in the list of most commonly reported drugs with high percentage of serious ADRs. The rest of the drugs in the list were all anti-infectives for systemic use. RR for a million boxes of consumption in this therapeutic group is not different from overall RR for a million boxes of drug consumption (Supplementary Table 1). However, the high reporting rate for these therapeutic groups may be due to higher consumption of these drugs. Additionally these agents mostly cause immediate and easily observable reactions defined under skin and subcutaneous tissue disorders and general disorders, and administration site conditions, for which causality between the drug and the reaction can easily be made. Accordingly, the percentage of serious ADRs reported with these drugs was relatively low in the database (Table 6).

\section{Conclusion}

This study showed that annual RRs in Turkey increased gradually in the 9-year study period. Major factors that might increase RR are: training activities conducted by TUFAM that raise awareness of pharmacovigilance, widening the scope of hospitals to all hospitals for 
employment of PvCPs, initiation of $\mathrm{PV}$ inspections in March 2010, authorization of a large number of biotechnological drugs and initiation of patient support programmes for these drugs. Though regulations that allow patient reporting became effective in April 2014 with the publication of "Regulation on safety of medicines", patient reports were started to be accepted by TUFAM in 2013. This is also a contributing factor for the increased rate of reporting in the study period.

Acknowledgments The authors gratefully acknowledge Professor Hakan Ergun (Ankara University Faculty of Medicine) for his helpful comments and advice on the manuscript.

\section{Compliance with Ethical Standards}

Conflict of interest Gulnihal Ozcan, Emel Aykac, Yelda Kasap, Nergiz Temiz Nemutlu, Ebru Sen and Nigar Demet Aydinkarahaliloglu have no conflicts of interest that are directly relevant to the content of this study.

Ethical approval Ethical approval was not required for the study.

Funding No sources of funding were used to assist in the preparation of this study.

Author contributions N. D. Aydinkarahaliloglu, E. Aykac and G. Ozcan designed the study. G. Ozcan analysed the data and wrote the manuscript. N. D. Aydinkarahaliloglu, E. Aykac, G. Ozcan, Y. Kasap, N. T. Nemutlu, and E. Sen collected the data. All authors saw and approved the final version of the manuscript.

Open Access This article is distributed under the terms of the Creative Commons Attribution-NonCommercial 4.0 International License (http://creativecommons.org/licenses/by-nc/4.0/), which permits any noncommercial use, distribution, and reproduction in any medium, provided you give appropriate credit to the original author(s) and the source, provide a link to the Creative Commons license, and indicate if changes were made.

\section{References}

1. Jönsson AK. Drug-related morbidity and mortality: pharmacoepidemiological aspects: Linköping, Sweden: Linköping University, Faculty of Health Sciences; 2007.

2. Pirmohamed M, James S, Meakin S, Green C, Scott AK, Walley TJ, Farrar K, Park BK, Breckenridge AM. Adverse drug reactions as cause of admission to hospital: prospective analysis of 18,820 patients. BMJ. 2004;329(7456):15-9.

3. Sultana J, Cutroneo P, Trifiro G. Clinical and economic burden of adverse drug reactions. J Pharmacol Pharmacother. 2013;4(Suppl 1):S73-7.

4. Bates DW, Spell N, Cullen DJ, Burdick E, Laird N, Petersen LA, Small SD, Sweitzer BJ, Leape LL. The costs of adverse drug events in hospitalized patients. JAMA. 1997;277(4):307-11.

5. WHO. Safety of medicines: a guide to detecting and reporting adverse drug reactions. Geneva: WHO; 2002.

6. WHO. The safety of medicines in public health programmes: pharmacovigilance an essential tool. Geneva: WHO; 2006.
7. Lindquist M. VigiBase, the WHO Global ICSR Database System: basic facts. Drug Inf J. 2008;42(5):409-19.

8. http://www.who-umc.org/DynPage.aspx ?id=98082\&mn1=7347\& $\mathrm{mn} 2=7252 \& m n 3=7322 \& m n 4=7326$. Accessed 17 Sep 2015.

9. Mazzitello C, Esposito S, De Francesco AE, Capuano A, Russo E, De Sarro G. Pharmacovigilance in Italy: an overview. J Pharmacol Pharmacother. 2013;4(Suppl1):S20-8.

10. Aagaard L, Strandell J, Melskens L, Petersen PS, Holme Hansen E. Global patterns of adverse drug reactions over a decade: analyses of spontaneous reports to VigiBase. Drug Saf. 2012;35(12):1171-82.

11. Biswas P. Pharmacovigilance in Asia. J Pharmacol Pharmacother. 2013;4(Suppl 1):S7-19.

12. Regulation on the monitoring and assessment of the safety of medicinal products for human use. http://www.resmigazete.gov. tr/eskiler/2005/03/20050322-7.htm. Accessed 7 Sep 2015.

13. Regulation on safety of medicines. http://www.resmigazete.gov. tr/eskiler/2014/04/20140415-6.htm. Accessed 7 Sep 2015.

14. Turkish Statistical Institute. Main statistics: population and demography. 2015. http://www.turkstat.gov.tr. Accessed 7 Sep 2015.

15. Turkey TMoHo. Health statistics yearbook 2013. Ankara: The Ministry of Health of Turkey; 2014.

16. http://www.meddra.org. Accessed 17 Sep 2015.

17. ICH harmonised tripartite guideline. Clinical safety data management: definitions and standards for expedited reporting E2A. http://www.ich.org/fileadmin/Public_Web_Site/ICH_Products/ Guidelines/Efficacy/E2A/Step4/E2A_Guideline.pdf. Accessed 17 Sep 2015.

18. Guidelines for ATC classification and DDD assignment 2013. http://www.whocc.no/filearchive/publications/1_2013guidelines. pdf. Accessed 11 May 2015.

19. Asseray N, Ballereau F, Trombert-Paviot B, Bouget J, Foucher N, Renaud B, Roulet L, Kierzek G, Armand-Perroux A, Potel G, Schmidt J, Carpentier F, Queneau P. Frequency and severity of adverse drug reactions due to self-medication: a cross-sectional multicentre survey in emergency departments. Drug Saf. 2013;36(12):1159-68.

20. Schmiedl S, Rottenkolber M, Hasford J, Rottenkolber D, Farker K, Drewelow B, Hippius M, Saljé K, Thürmann P. Self-medication with over-the-counter and prescribed drugs causing adverse-drug-reaction-related hospital admissions: results of a prospective, long-term multi-centre study. Drug Saf. 2014;37(4):225-35.

21. Martin RM, Biswas PN, Freemantle SN, Pearce GL, Mann RD. Age and sex distribution of suspected adverse drug reactions to newly marketed drugs in general practice in England: analysis of 48 cohort studies. Br J Clin Pharmacol. 1998;46:505-11.

22. Zopf Y, Rabe C, Neubert A, Gabmann KG, Rascher W, Hahn EG, Dormann H. Women encounter ADRs more often than do men. Eur J Clin Pharmacol. 2008;64:999-1004.

23. Fattinger K, Roos M, Vergeres P, Holenstein C, Kind B, Masche U, Stocker DN, Braunschweig S, Kullak-Ublick GA, Galeazzi RL, Follath F, Gasser T, Meier PJ. Epidemiology of drug exposure and adverse drug reactions in two swiss departments of internal medicine. Br J Clin Pharmacol. 2000;49:158-67.

24. Routledge PA, O’Mahony MS, Woodhouse KW. Adverse drug reactions in elderly patients. $\mathrm{Br} \mathrm{J}$ Clin Pharmacol. 2004;57(2):121-6.

25. Hemminki E, Gissler M, Toukomaa H. Exposure to female hormone drugs during pregnancy: effect on malformations and cancer. Br J Cancer. 1999;80(7):1092-7.

26. Kim MR, Qazi QH, Anderson VM, Valencia GB. A genetic male infant with female phenotype in camptomelic syndrome: a relationship to exposure to oral contraceptives during pregnancy. Am J Obstet Gynecol. 1995;172:1042-3. 
27. Hersh JH, Danhauer DE, Hand ME, Weisskopf B. Retinoic acid embryopathy: timing of exposure and effects on fetal development. JAMA. 1985;254:909-10.

28. Benke PJ. The isotretinoin teratogen syndrome. JAMA. 1984;251:3267-9.

29. Braun JT, Franciosi RA, Mastri AR, Drake RM, O’Neil BL. Isotretinoin dysmorphic syndrome. Lancet. 1984;1:506-7.

30. Product information: SPRYCEL(R) oral tablets, dasatinib oral tablets. Istanbul: Bristol-Myers Squibb Company (per manufacturer); 2015.

31. Gu Y, Ru T, Zhou YH, Hu Y. Adefovir as a possible teratogen: evidence from paternal exposure. Dig Liver Dis. 2014;46(12):1134-5.

32. Product information: ZYPREXA(R) solution for IM injection, oral tablets, orally disintegrating tablets, olanzapine solution for
IM injection, oral tablets, orally disintegrating tablets. Istanbul: Eli Lilly and Company; 2013.

33. Newport DJ, Calamaras MR, DeVane CL, Donovan J, Beach AJ, Winn S, Knight BT, Gibson BB, Viguera AC, Owens MJ, Nemeroff CB, Stowe ZN. Atypical antipsychotic administration during late pregnancy: placental passage and obstetrical outcomes. Am J Psychiatry. 2007;164(8):1214-20.

34. Kulkarni J, Storch A, Baraniuk A, Gilbert H, Gavrilidis E, Worsley R. Antipsychotic use in pregnancy. Expert Opin Pharmacother. 2015;16(9):1335-45.

35. Spyropoulou AC, Zervas IM, Soldatos CR. Hip dysplasia following a case of olanzapine exposed pregnancy: a questionable association. Arch Womens Ment Health. 2006;9(4):219-22. 